\title{
Left Pulmonary Artery Agenesis in a Pediatric Patient - Case Report
}

\author{
Cristina Blesneac', Carmen Corina Șuteu', Marian Pop², Rodica Togănel' \\ 1 University of Medicine and Pharmacy, Tîrgu Mureș, Romania \\ 2 County Emergency Clinical Hospital, Tîrgu Mureș, Romania
}

\section{CORRESPONDENCE \\ Carmen Corina Șuteu \\ 38 Gheorghe Marinescu St \\ 540139 Tîrgu Mureș, Romania \\ Tel: 0265215551 \\ Email: suteucarmen@yahoo.com}

\section{ARTICLE HISTORY}

Received: 15 April, 2016

Accepted: 18 May, 2016
Cristina Blesneac • University of Medicine and Pharmacy, 38 Gheorghe Marinescu St, 540139, Tîrgu Mureș, Romania, Tel: +40 265215551

Marian Pop • County Clinical Emergency Hospital, 50 Gheorghe Marinescu St, 540000 Tîrgu Mureș, Romania, Tel: +40 265211292

Rodica Togănel • University of Medicine and Pharmacy, 38 Gheorghe Marinescu St, 540139, Tîrgu Mures, Romania, Tel: +40 265215551

\begin{abstract}
Unilateral pulmonary artery agenesis is a rare congenital anomaly, that may develop in isolation, or in association with other congenital cardiovascular anomalies, such as tetralogy of Fallot, septal defects, right-sided aortic arch, or pulmonary atresia. Left-sided pulmonary artery agenesis is less frequent than the right-sided one. Diagnosis of unilateral pulmonary artery agenesis can be difficult. We report the case of a 15 year-old boy who presented with reduced exercise tolerance, shortness of breath and cyanosis. He was diagnosed with left pulmonary artery agenesis, associated with subaortic-ventricular septal defect, right-sided aortic arch, and severe pulmonary arterial hypertension (PAH), that precluded the surgical repair. Pulmonary vasodilator therapy was initiated in this case. The mortality rate of this rare anomaly is high due to its complications. It is essential to establish an early and correct diagnosis, in order to provide adequate treatment and prevent complications in this disease.
\end{abstract}

Keywords: pulmonary artery, congenital heart disease, pulmonary hypertension

\section{INTRODUCTION}

Unilateral pulmonary artery agenesis (UPAA) is a rare congenital anomaly, frequently diagnosed during adolescence, ${ }^{1}$ and usually is associated with other cardiac congenital anomalies, such as tetralogy of Fallot, atrial and/or ventricular septal defect, coarctation of the aorta, right-sided aortic arch, truncus arteriosus, and pulmonary atresia. ${ }^{1,2}$ It can occur as a single disorder also. ${ }^{1}$ In cases of isolated UPAA, the reported incidence ranges between 1 in 200,000 to 1 in $300,000 .{ }^{3}$ Right-sided UPAA is more frequent than the left-sided one. ${ }^{2}$ Left-sided agenesis is more likely to occur in association with other congenital cardiovascular anomalies, time of presentation in such cases being at a younger age. ${ }^{1,4}$ In case of isolated UPAA, patients may be asymptomatic or they manifest only minor symptoms, therefore surviving into adulthood. ${ }^{1}$ Late diagnosis is possible in such cases, usually after an abnormal chest radiograph. ${ }^{5}$

We report the case of a unilateral pulmonary agenesis in a young child, associated with ventricular septal defect, right aortic arch and pulmonary arterial hypertension. 


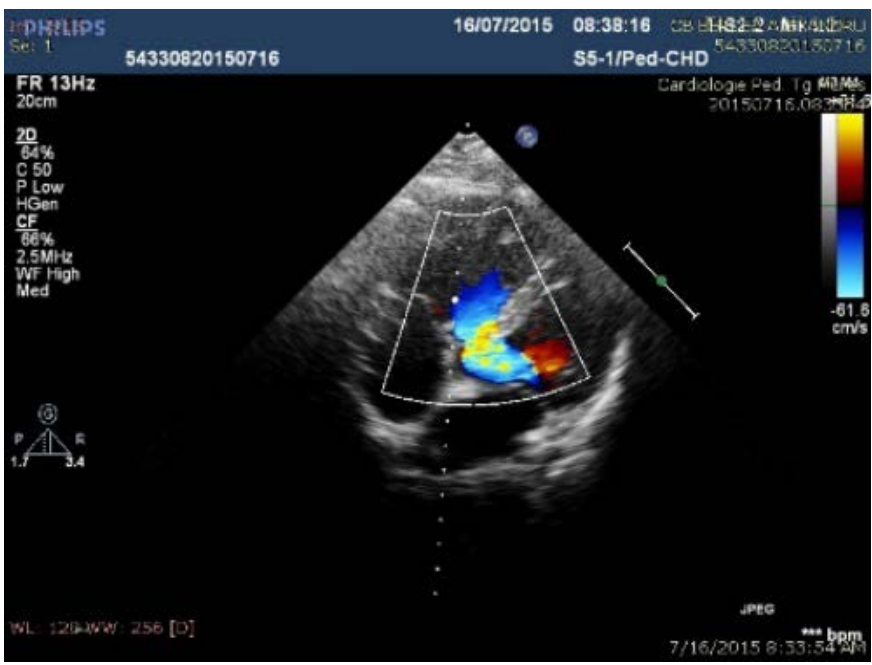

FIGURE 1. Transthoracic color Doppler echocardiography, apical 5-chamber view, showing the ventricular septal defect, with the right-to-left component of the shunt.

\section{CASE REPORT}

We report the case of a 15 year-old boy, who presented in our department for reduced exercise tolerance, shortness of breath and cyanosis. From his personal background we found out that he has been diagnosed with ventricular septal defect since infancy, but for personal reasons, he has not been evaluated since then in a pediatric cardiology department.

At the time of admittance, he presented with cyanosis, with room air $\mathrm{O}_{2}$ saturation of $80 \%$, an enlarged chest diameter, a dorsal scoliosis, a normal first and accentuated second heart sound, a grade $3 / 6$ pansystolic murmur all

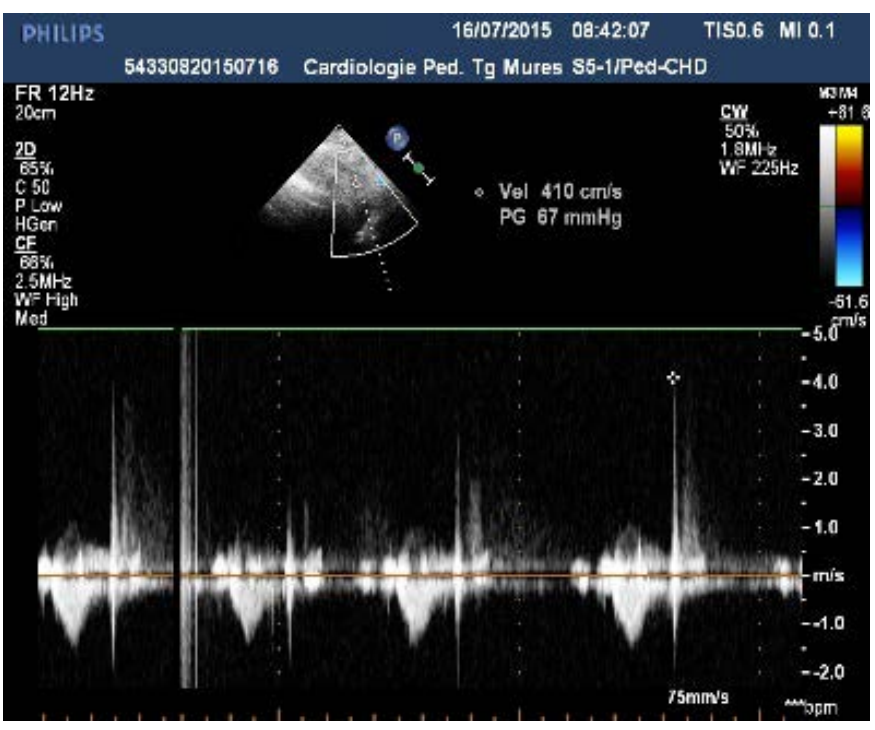

FIGURE 3. The estimated mean pulmonary arterial pressure based on the pulmonary regurgitation jet.

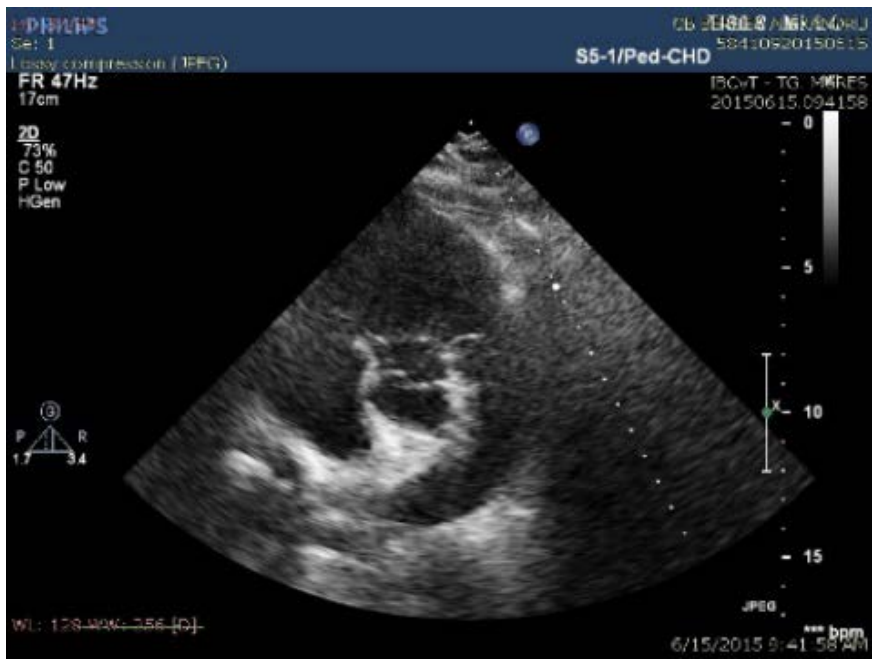

FIGURE 2. Transthoracic 2D echocardiography, parasternal short axis view, showing the ventricular septal defect, dilated main pulmonary artery, and agenesis of the left pulmonary artery.

over the precordial area. Lung auscultation revealed decreased respiratory sounds on the left hemithorax, the rest of the clinical examination was normal.

A six-minute walk test was performed, the walked distance being $432 \mathrm{~m}$, with desaturation from $80 \%$ to $57 \%$ with room air.

Laboratory results showed polycythemia (red blood cells $\left.6.65 \times 10^{6} / \mu \mathrm{l}\right)$, an increased hemoglobin level $(19 \mathrm{~g} / \mathrm{dl})$, and a brain natriuretic peptide (BNP) level of $4.9 \mathrm{pg} / \mathrm{ml}$, with no remarkable changes in the other test results.

Chest $\mathrm{X}$-ray revealed a volume loss of the left lung, with a shift of the mediastinal structures to the left, small left apical pneumothorax, hyperinflation of the right lung, right sided aortic arch, and a cardio-thoracic ratio of 0.44 .

Echocardiography showed a large $(15 \mathrm{~mm})$, non-restrictive, posterior mal alignment type subaortic ventricular septal defect, with bidirectional shunt, dilated right cardiac chambers, a dilated main pulmonary artery with no visualization of the left pulmonary artery, with severe pulmonary arterial hypertension (mean pulmonary artery pressure of $67 \mathrm{mmHg}$ ), and a right aortic arch (Figures 1, 2 and 3).

A computer tomography (CT) was also performed, showing a right-left thoraco-pulmonary asymmetry, with left lung hypoplasia, due to left pulmonary artery agenesis, with shifting of the mediastinal structures to the left, a 10 $\mathrm{mm}$ bubble of emphysema in the postero-superior area of the left lung, a large subaortic ventricular septal defect, a right aortic arch, a dilated main pulmonary artery, a left pulmonary artery agenesis, the blood supply to the left lung being provided by aorto-to-pulmonary collaterals (Figures 4 and 5). 


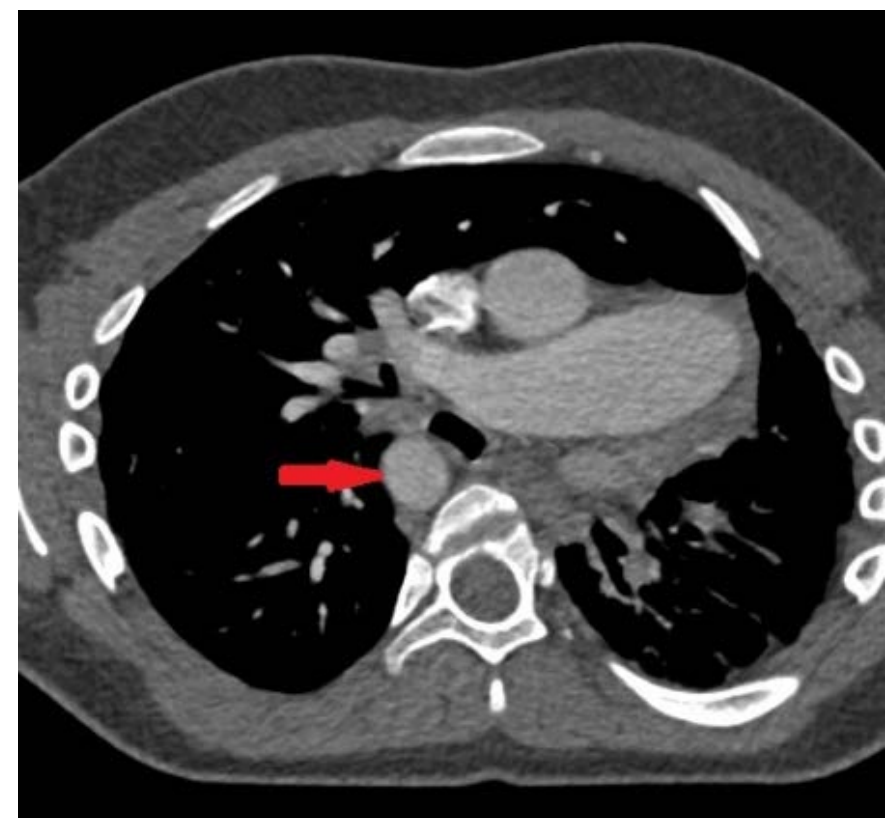

FIGURE 4. CT angiography, axial image. The pulmonary trunk emerges from the right ventricular outflow track, and gives rise to a single right pulmonary artery. A right-sided aortic arch is noted (red arrow). The left lung is hypoplastic.

Cardiac catheterization confirmed the absence of the left pulmonary artery and demonstrated an indexed pulmonary vascular resistance in the room air of $20.92 \mathrm{Wood}$ units, decreasing to 16.66 Wood units on $100 \%$ oxygen, and to 16.65 Wood units on $100 \%$ oxygen + nitric oxide. It also revealed large aorto-pulmonary collaterals supplying the left lung circulation.

Pulmonary vasodilator treatment with a dual endothelin-receptor antagonist was initiated, with a starting dose of $62.5 \mathrm{mg}$ twice a day, and after 4 weeks it was increased to a maximum dose of $125 \mathrm{mg}$ twice a day.

During the 6-month follow-up, after initiating pulmonary vasodilator treatment, the patient's exercise tolerance remained poor, but a mild improvement of the 6-minute walking test was noted. Echocardiography showed no significant changes between the initial and the 6-month follow-up parameters.

\section{DISCUSSION}

Unilateral agenesis of the pulmonary artery was first described by Fraentzel in 1868.6 It is a rare congenital anomaly with an estimated prevalence of 1 in $200,000^{5}$ and occurs due to a malformation of the sixth aortic arch of the affected side during embryogenesis. ${ }^{7}$

Studies showed that left-sided agenesis occurs half as frequent as the right-sided ones, ${ }^{8}$ with $80 \%$ of cases being

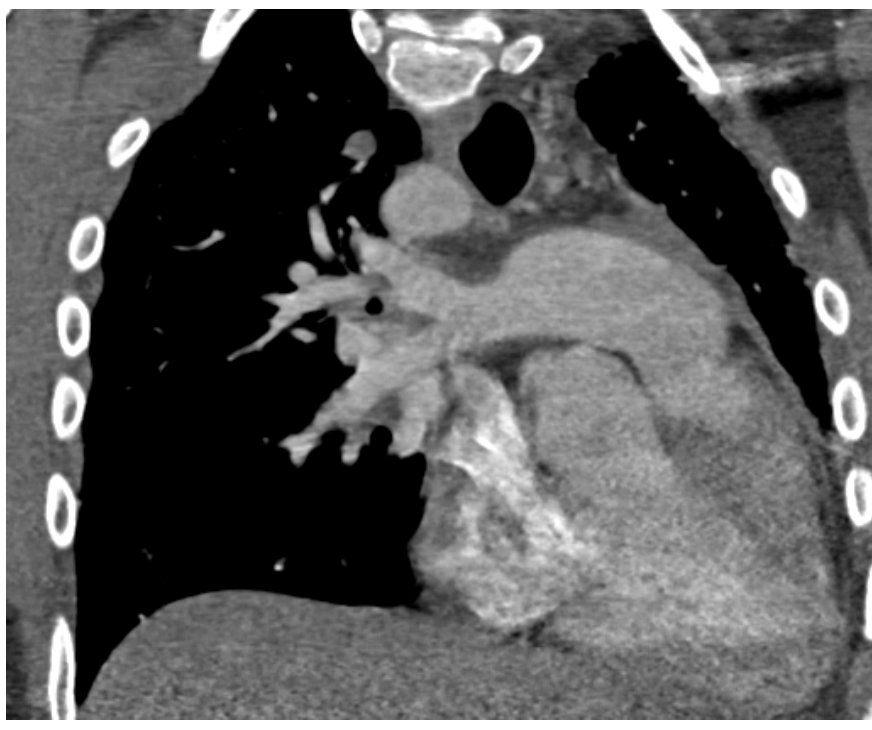

FIGURE 5. CT angiography, coronal image. No left pulmonary artery visualized. There is right-left thoraco-pulmonary asymmetry, with left lung hypoplasia, and a shift of the mediastinal structures to the left.

associated with a congenital cardiovascular malformation, thus being diagnosed early in life. ${ }^{3}$ In the case of our patient, left pulmonary artery agenesis occured in association with a ventricular septal defect and a right-sided aortic arch, and the complete diagnosis was made at the age of 15 years, when he first presented in our department.

Diagnosis of UPAA can be difficult, but a chest radiograph can provide important clues: an asymmetric lung field, with an ipsilateral small hemithorax holding a hyperlucent lung. 5,9 In our patient, the chest X-ray revealed a volume loss of the left lung, with a shift of the mediastinal structures to the left, small left apical pneumothorax, increased pulmonary vascular markings, right sided aortic arch. The exact diagnosis can be made by echocardiography, CT, or magnetic resonance imaging, with pulmonary angiography being considered the gold standard for diagnosis. ${ }^{10}$ Our patient was diagnosed by echocardiography, and it was confirmed by CT and angiography.

Regarding the symptoms, studies have shown that approximately $30 \%$ of patients with UPAA may remain asymptomatic during their lifetime, the other $70 \%$ can present with serious pulmonary hypertension and congestive heart failure. ${ }^{1,11}$ The most frequently encountered symptoms are recurrent lung infections (37\%), decreased exercise tolerance, dyspnea (40\%), and hemoptysis (1820\%). ${ }^{1,12}$ Our patient presented with reduced exercise tolerance, shortness of breath and cyanosis.

Pulmonary arterial hypertension (PAH) is a severe complication of UPAA that affects outcome, with a report- 
ed mortality rate in of $44 \% .{ }^{1} \mathrm{PAH}$ may result from blood flow directed only to the remaining pulmonary artery; increased blood flow leads to sheer stress on the endothelium, and releasing of vasoconstrictors. ${ }^{10}$ Chronic vasoconstriction of the pulmonary arterioles leads to remodeling, which results in an increase in pulmonary vascular resistance, and pulmonary arterial hypertension. ${ }^{10}$ In the case of our patient, right heart catheterization revealed severe pulmonary arterial hypertension, with extremely high pulmonary vascular resistances.

The treatment for UPAA depends on age at presentation, symptoms of the patient, associated cardiovascular anomalies, and PAH. ${ }^{3,8}$ The options include revascularization of the distal affected pulmonary artery, considering that intrapulmonary arteries are still formed during embryological development. ${ }^{1,3,8}$ In older patients, because the intrapulmonary arteries can be severely narrowed or even completely obstructed by fibrosis, revascularization is not encouraged or even feasible. ${ }^{13,14}$ In our patient, late presentation and severe PAH precluded any surgical intervention. Patients with recurrent infections or massive hemoptysis may need pneumonectomy or embolization of the affected vessels. ${ }^{3}$ With PAH remaining a severe complication of UPAA, pulmonary vasodilator therapy with endothelin-receptor antagonist may be considered as a long-term therapy and it may improve survival. ${ }^{3}$ In case of our patient, a dual endothelin-receptor antagonist was initiated, with a mild improvement of the 6-minute walk test, but no significant changes in the echocardiographic parameters.

The mortality rate of this rare anomaly is high because of its complications. The overall mortality rate in UPAA is approximately $7 \%,{ }^{1}$ with right heart failure, respiratory failure, massive pulmonary hemorrhage being the most common causes of death. ${ }^{1,15,16}$

\section{CONCLUSION}

Unilateral pulmonary artery agenesis is a rare congenital malformation, and diagnosis is very important because of its severe complications that can lead to mortality. We reported the case of a 15 year-old boy with left pulmonary artery agenesis, associated with ventricular septal defect, and right-sided aortic arch, who developed severe PAH that did not allow surgical repair, and in whom pulmonary vasodilator therapy was initiated. It is essential to establish an early and correct diagnosis, in order to provide adequate treatment and prevent complications in this disease.

\section{CONFLICT OF INTEREST}

Nothing to declare.

\section{REFERENCES}

1. Ten Harkel AD, Blom NA, Ottenkamp J. Isolated unilateral absence of a pulmonary artery: a case report and review of the literature. Chest. 2002:122:1471-1477.

2. Moosavi SAJ, Iranpour A. Unilateral pulmonary artery agenesis in an adult patient with coughand hemoptysis: a case report. Tanaffos. 2014;13(4):5860.

3. Kruzliak P, Syamasundar RP, Novak M, Pechanova O, Kovacova G. Unilateral absence of pulmonary artery: pathophysiology, symptoms, diagnosis and current treatment. Arch Cardiovasc Dis. 2013;106:448-454

4. Ghanbari H, Feldman D, David S, Saba S. Unilateral absence of a left pulmonary artery: successful therapeutic response to a combination of bosentan and warfarin. Circ Cardiovasc Imaging. 2009;2:e46-e48.

5. Bouros D, Pare P, Panagou P, Tsintiris K, Siafakas N. The varied manifestation of pulmonary artery agenesis in adulthood. Chest. 1995;108:670-676.

6. Fraentzel O. Angeborener defekt der rechten lungenarterie. Virchows Arch. Pathol Anat. 1868;43:420.

7. Emren VS, Tülüce SY, Tülüce K. Isolated congenital unilateral agenesis of the left pulmonary artery with left lung hypoplasia in an asymptomatic adult patient. Acta Cardiol Sin. 2015;31:572-575

8. Flores $M$, Letter $H$, Derrick $E$, et al. Unilateral Absence of the Left Pulmonary Artery With an Associated Vascular Anomaly in Adulthood. Cureus. 2016;8(3):e527.

9. Griffin N, Mansfield L, Redmond KC, et al. Imaging features of isolated unilateral pulmonary artery agenesis presenting in adulthood: a review of four cases. Clin Radiol. 2007;62(3):238-244

10. Purani C, Mehariya KM, Gupta K, Patel P. Hypoplasia of left pulmonary artery: A rare congenital heart disease. Indian J Child Health. 2015;2(1):3133.

11. Toews WH, Pappas G. Surgical management of absent right pulmonary artery with associated pulmonary hypertension. Chest. 1983;84:497-499.

12. Shakibi JG, Rastan H, Nazarian I, Paydar M, Aryanpour I, Siassi B. Isolated unilateral absence of the pulmonary artery. Review of the world literature and guidelines for surgical repair. Jpn Heart J. 1978;19:439-451.

13. Pool PE, Vogel JH, Blount Jr SG. Congenital unilateral absence of a pulmonary artery.The importance of flow in pulmonary hypertension. Am J Cardiol. 1962;10:706-32.

14. Mehta AC, Livingston DR, Kawalek W, et al. Pulmonary artery agenesis presenting as massive hemoptysis - a case report. Angiology. 1987;38:6771.

15. Thompson JW, Nguyen CD, Lazar RH, et al. Evaluation and management of hemoptysis in infants and children. A report of nine cases. Ann Otol Rhinol Laryngol. 1996;105:516-520.

16. Ferrari M, Karrazi R, Lampronti G, et al. Effect of changing position on arterial oxygenation in a patient with agenesia of the left pulmonary artery. Respiration. 1997;64:371-374. 